\title{
REDUCED ORDER MODEL FOR TRANSFORMER TRANSIENTS
}

\author{
Francisco de Leon Adam Semlyen \\ Department of Electrical Engineering \\ University of Toronto \\ Toronto, Ontario, Canada, M5S 1A4
}

\begin{abstract}
A complete model for transformers is derived on the base of very efficiently calculated elementary (tum-to-tum) parameters. A high-order tum-to-tum model is constructed for the windings. This model is reduced to a lower order by operating on the resulting matrices. An electric equivalent circuit for the core is obtained from the principle of duality. By the use of test tums the winding model is interfaced with the iron-core. For validation, the frequency response of the model has been compared with test results. The model for the calculation of transients has the form of a Norton equivalent circuit and it can easily be incorporated in a power system transients program such as the EMTP. Examples of calculated transients are given for illustration and further validation.
\end{abstract}

Keywords: Transformer modeling, Electromagnetic transients.

\section{INTRODUCTION}

The progress in transformer modeling for the calculation of electromagnetic transients has not kept pace with the advances in the modeling of most other major power system components. This is probably due to the complexity of the physical phenomena that take place in the transformer. There has been much work reported in the literature but no power transformer model for a wide range of frequencies is yet available. In this paper we present a model for the transformer windings that is suitable for a wide frequency range. In reference [1] we computed the parameters (leakage inductances and capacitances) in a very efficient way on a tum-to-turn basis. Now we use that information to form a model for the windings. Then we combine the windings and iron-core models to form a complete transformer model.

There are three main approaches followed in the modeling of transformers:

a) Modeling based on the principle of duality. In references [2] and

[3] it was shown that an equivalent electric circuit for a transformer can be derived from its magnetic circuit by applying the principle of duality. Therefore, the leakage flux (rather than leakage inductance) is used for modeling the phenomena in the air. This may lead to inaccurate terminal response, which depends on the leakage inductance and not on the leakage flux. This problem was solved by the use of negative inductances; see references [4] and [5]. The parameters of these (duality based) models are calculated assuming that the magnetic field is in axial direction in cylindrical geometry. As shown in reference [1], this leads to a greater error than our proposed image method when predicting the terminal leakage inductance. However, a model based on the principle of duality reflects properly the flux balance and thus the nonlinear iron-core can be represented accurately. Recently, this approach has been used in the modeling of transformers in highly saturated conditions; see reference [6]

91 WM 126-3 PWRD A paper recommended and approved by the IEEE Transformers Committee of the IEEE Power Engineering Society for presentation at the IEEE/PES 1991 Winter Meeting, New York, New York, February 3-7, 1991. Manuscript submitted August 31,1990 ; made available for printing November 30, 1990. b) Modeling based on leakage inductances. These models frequently use an inverse inductance matrix, often referred to as $\Gamma$ [7],[11]. $\Gamma$ models reflect accurately the transfer characteristics of the transformer (short circuit response) because their parameters are obtained from short circuit tests. As we will show later in this paper, there is no need to use the $\boldsymbol{\Gamma}$ matrix to construct a model from leakage inductance information. The $\Gamma$ models present a difficulty in relation with the iron-core which has to be attached in a heuristic way since the magnetizing effects are lost in the short circuit tests.

c) Modeling based on self and mutual inductances. There exist accurate formulae to compute the self and mutual inductances for sections or windings of a transformer $[12,13]$. However, because of the presence of the iron-core, the self and mutual inductances have values that are very close in magnitude, so that this approach leads to an ill-conditioned set of equations.

We use a combination of the first two approaches: leakage inductances (tum-to-turn) are used for the modeling of the windings and the principle of duality is used for the iron-core.

A model considering each tum as an independent entity would be impractical due to its large size. To overcome this problem, we lump, by matrix manipulations, as many tums as the frequency range we are interested in permits. We assume for the calculation of the winding inductance matrix that the geometry is axisymmetrical and that the ironcore has infinite permeability. The magnetizing effects are then included into the model by the use of test turns and the principle of duality.

\section{HIGH-ORDER WINDING MODEL}

In reference [1] we have calculated the parameters taking the turns as basic elements for the capacitances and the leakage inductances. For the calculation of the capacitances we have used the charge simulation method with axisymmetric geometry. We have used the image method for the calculation of the turn leakage inductances, based on axisymmetric geometry. As a consequence, our model for the windings is not able to represent the mutual leakage effects between different legs of a transformer; it accounts only for the mutual effects between the tums wound on the same leg. The inductive coupling through the leakage paths (between two different legs) is believed to be negligible, especially when it is compared with the tight coupling from the yokes. However, the capacitive coupling between the windings on different legs and to the tank may not be negligible. We can add extemal capacitance to represent this effect after the leg-by-leg model is constructed.

\section{Inductive Model}

For an $N$ turn transformer the voltage-current governing equation for the inductive part of the transformer (using leakage inductances, equation (17) from [1]), is

$$
\mathrm{v}=\mathrm{L}^{\prime} \frac{d}{d t} \mathrm{i}
$$

The order of the matrix $\mathbf{L}^{\prime}$ is $N-1$ because one of the turns serves as 'reference.

Relation (1) constitutes the backbone of the inductive model for windings. A similar process for obtaining a model was described in [10] for complete coils and it involves the inversion of the matrix $\mathbf{L}^{\prime}$ to get an inverse inductance matrix $\Gamma^{\prime}$. As we will see, obtaining the $\Gamma^{\prime}$ matrix is not necessary since we can integrate equation (1) and get a Norton equivalent directly. We will lump several turns in series before integrating in order to reduce the model to a manageable size. 


\section{Capacitive Model}

The turn capacitance matrix $\mathbf{C}^{\mathbf{t u r n}}$, obtained in reference [1] from the charge simulation method, relates currents and voltages through the turn-to-turn capacitances. We can use this information to produce a nodal model. Shifting half of each capacitance to the two ends of the turn, we can write the nodal equation

$$
\mathrm{i}_{C \text { node }}=\mathrm{C}_{\text {node }} \frac{d}{d t} \mathbf{v}_{\text {node }}
$$

where the nodal capacitance matrix $\mathbf{C}_{\text {node }}$ can be obtained from the turn capacitance matrix $\mathrm{C}^{\text {turn }}$ by recognizing which elements are connected to each node. The resulting matrix $\mathrm{C}_{\text {node }}$ is of order $2 \mathrm{~N}$, with the general terms given by

$$
\begin{gathered}
C_{2 i-1,2 j-1}=C_{2 i, 2 j}=1 / 2 C_{i, j}^{\text {turn }} \\
C_{2 i-1,2 j}=C_{2 i, 2 j-1}=0
\end{gathered}
$$

Figure 1 shows an equivalent circuit for the winding model. The inductances are not explicitly shown. This model is of a very high order since it is based on turm-to-turn variables.

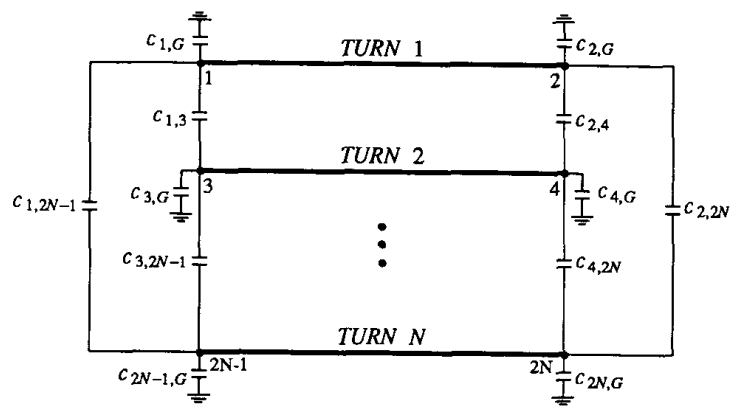

Figure 1. Winding model

\section{MODEL REDUCTION}

To reduce the model, we lump several series connected tums to form sections. The essence of the process is the shifting of some (internal) capacitances towards pre-established section nodes. Once the capacitances are moved to section nodes, the loop inductances are easily lumped. In order to show how the reduction is done, a 3-tum section will be formed step by step; see Figures 2a to $2 \mathrm{e}$.

\section{Reduction of Capacitances}

The first step is to move the capacitances connected to internal nodes to the external ones (section nodes). For convenience, an odd number of tums per section is chosen. The reduction of the capacitance network can be done by operating on the nodal capacitance matrix $\mathbf{C}_{\text {node }}$. Consider first that the three turns are disconnected (Figure 2a). The nodal capacitance matrix for this arrangement is

$\mathrm{C}_{\text {rose }}=\left[\begin{array}{cccccc}c_{16}+c_{13}+c_{15} & 0 & -c_{13} & 0 & -c_{15} & 0 \\ 0 & c_{26}+c_{24}+c_{26} & 0 & -c_{24} & 0 & -c_{26} \\ -c_{13} & 0 & c_{36}+c_{13}+c_{33} & 0 & -c_{35} & 0 \\ 0 & -c_{24} & 0 & c_{46}+c_{24}+c_{46} & 0 & -c_{46} \\ -c_{15} & 0 & -c_{35} & 0 & c_{56}+c_{15}+c_{35} & 0 \\ 0 & -c_{26} & 0 & -c_{46} & 0 & c_{66}+c_{26}+c_{46}\end{array}\right]$

Lower case $c$ is used for capacitances in the circuit; upper case $C$ for the elements in the matrix. For example, $C_{11}=c_{1 G}+c_{13}+c_{15}$ and $C_{13}=-c_{13}$. Connecting the end of one turn to the beginning of the next one, gives the circuit shown in Figure $2 \mathrm{~b}$. By moving the capacitances connected to the internal nodes (2-3 and 4-5) towards the nearest extemal node $(1$ or 6 ), we obtain the circuit shown in Figure $2 \mathrm{c}$. This circuit has the section capacitance matrix

$$
\mathrm{C}_{\text {node }}^{\text {sec }}=\left[\begin{array}{cc}
c_{1 G}+c_{2 G}+c_{3 G}+c_{15}+c_{24}+c_{26}+c_{35} & -c_{15}-c_{24}-c_{26}-c_{35} \\
-c_{15}-c_{24}-c_{25}-c_{35} & c_{4 G}+c_{5 G}+c_{6 G}+c_{15}+c_{24}+c_{26}+c_{35}
\end{array}\right]
$$

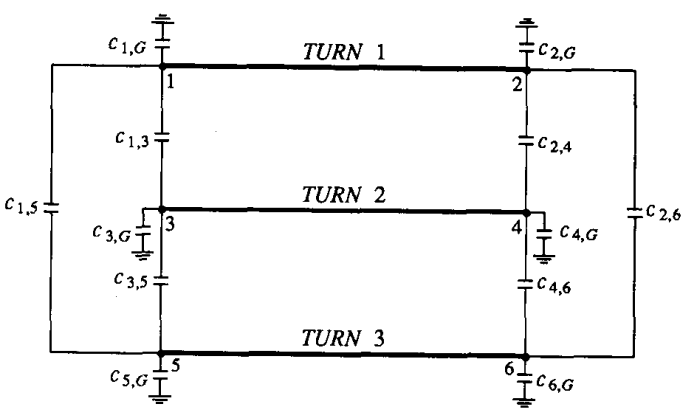

Figure 2a. Turn-to-turn model (turns disconnected)

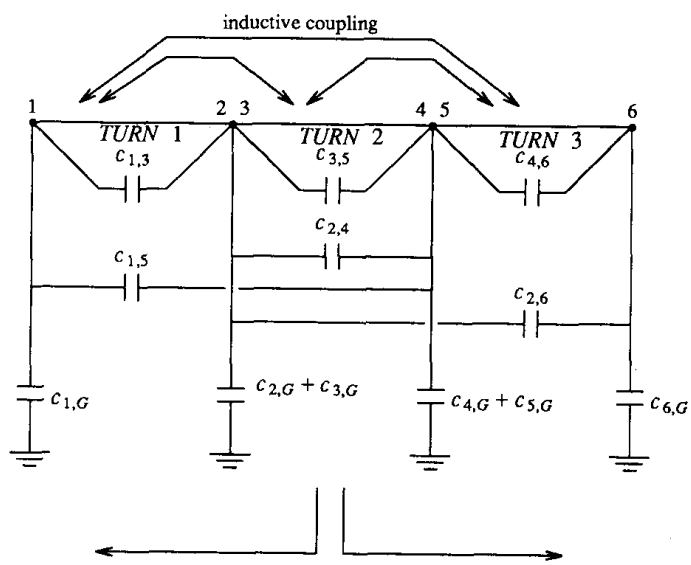

Figure 2b. First lumping step

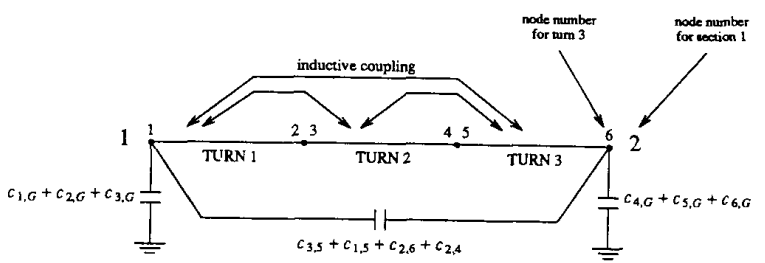

Figure 2c. Capacitances moved to the section nodes

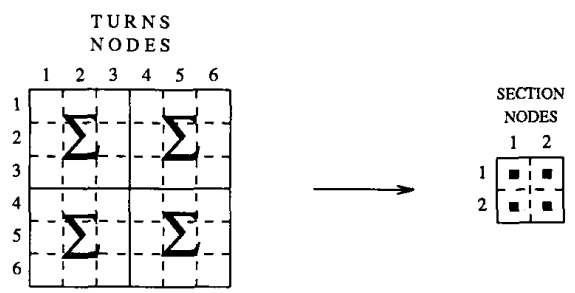

Figure 2d. Reduction process

Note that we can obtain this matrix by operating on the $\mathbf{C}_{\text {node }}$ matrix (equation (4)): partitioning $\mathbf{C}_{\text {node }}$ in four square matrices of order 3 and adding all the elements in each submatrix, we obtain the capacitance matrix (5) of the reduced model (see Figure 2d). In terms of the elements of the matrix $\mathbf{C}_{\text {node }}$, the last equation can be written as

$$
\mathrm{C}_{\text {node }}^{\text {sec }}=\left[\begin{array}{ll}
C_{11}+C_{22}+C_{33}+2 C_{13} & C_{15}+C_{24}+C_{26}+C_{35} \\
C_{15}+C_{24}+C_{26}+C_{35} & C_{44}+C_{55}+C_{66}+2 C_{46}
\end{array}\right]
$$


There is no need to write explicitly the matrix $\mathrm{C}_{\text {node }}$ of order $2 \mathrm{~N}$ since all the needed information is contained in the matrix $\mathrm{C}^{t \text { urn }}$ which is of order $N$. For our example, using equation (3a) and the definitions of self $\left(C_{i i}\right)$ and mutual $\left(C_{i j}, i \neq j\right)$ capacitances in equation (6), we have

$$
\begin{gathered}
C_{1,1}^{\text {sec }}=C_{1,1}^{\text {turn }}+1 / 2\left(C_{1,2}^{\text {turn }}+C_{2,1}^{\text {turn }}\right)+1 / 2 C_{2,2}^{\text {turn }} \\
C_{2,2}^{\text {sec }}=C_{3,3}^{\text {turn }}+1 / 2\left(C_{3,2}^{\text {turn }}+C_{2,3}^{\text {turn }}\right)+1 / 2 C_{2,2}^{\text {turn }} \\
C_{1,2}^{\text {sec }}=C_{2,1}^{\text {sec }}=C_{1,2}^{\text {turn }}+1 / 2\left(C_{1,2}^{\text {turn }}+C_{2,1}^{\text {turn }}\right)
\end{gathered}
$$

The procedure described above can be generalized for any (odd) number of turns per section and any number of sections per winding. The general equations to form a winding are

$$
\begin{gathered}
C_{i i \text { node }}^{\text {sec }}=\sum_{i=i_{i}}^{l_{i}} \sum_{j=i_{j}}^{l_{i}} C_{i j}^{\text {turn }}+1 / 2 \sum_{i=i_{i}}^{l_{i}} C_{i, m}^{t u r n}+1 / 2 \sum_{j=i_{i}}^{l_{i}} C_{m, j}^{\text {turn }}+1 / 2 C_{m, m}^{\text {turn }} \\
C_{i j \text { node }}^{\text {sec }}=\sum_{i=i_{i}}^{l_{i}} \sum_{j=i_{j}}^{l_{j}} C_{i j}^{\text {turn }}+1 / 2 \sum_{i=i_{i}}^{l_{i}} C_{i, m}^{t u r n}+1 / 2 \sum_{j=i_{j}}^{l_{j}} C_{m, j}^{\text {turn }}
\end{gathered}
$$

where

$$
\begin{aligned}
l_{i} & =i_{i}+\left(w_{k}-1\right) / 2-1 \\
w_{k} & =\text { number of lumped tums in section } k \\
i_{i} & =\text { first tum in section } i \\
m & =\text { tum in the center of this section }
\end{aligned}
$$

From equations (7a) and (7b) we can see that the reduction process consists simply of an addition of elements in the tum capacitance matrix $\mathrm{C}^{\text {twrn }}$ by blocks. The boundaries for forming the blocks are the turns located at the beginning, at the center, and at the end of the section to be formed. Each section produces four entries in the node capacitance matrix for sections $\mathrm{C}_{\text {node }}^{\text {sec }}$. The procedure is presented schematically in Figure 2e.

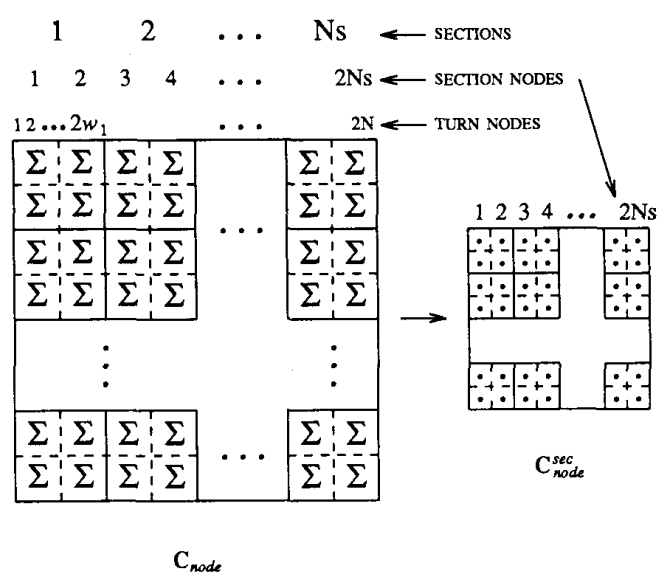

Figure 2e. General reduction procedure for the capacitive network

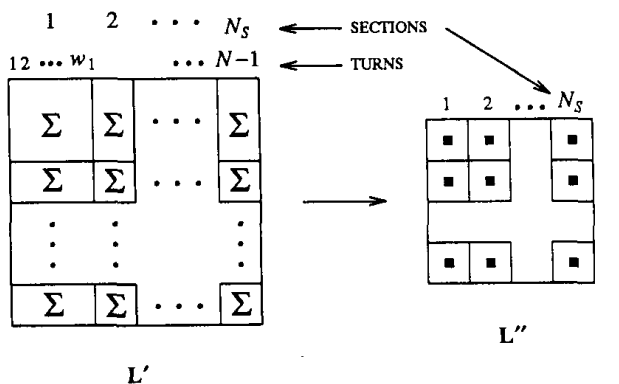

Figure 3. General reduction procedure for the loop inductance matrix

\section{Reduction of Inductances}

Once the capacitances have been moved to the ends of the section, the turns inside each section become connected in series (see Figure $2 \mathrm{c}$ ). Then, for a section containing $k+1$ turns, we have

$$
i_{i}=i_{i+1}=i_{i+2}=\cdots=i_{i+k}
$$

Applying this condition to equation (1), we see that the equivalent inductance is the sum of all elements (self and mutual). In our example $i_{1}=i_{2}=i_{3}$ and equation (1) with turn $N$ as reference becomes

$$
\left[\begin{array}{l}
v_{1}-v_{N} \\
v_{2}-v_{N} \\
v_{3}-v_{N}
\end{array}\right]=\left[\begin{array}{lll}
L_{11}^{\prime} & L_{12}^{\prime} & L_{13}^{\prime} \\
L_{21}^{\prime} & L_{22}^{\prime} & L_{23}^{\prime} \\
L_{31}^{\prime} & L_{32}^{\prime} & L_{33}^{\prime}
\end{array}\right] \frac{d}{d t}\left[\begin{array}{l}
i_{1} \\
i_{1} \\
i_{1}
\end{array}\right]
$$

Adding the three rows, yields

$$
v_{1}+v_{2}+v_{3}-3 v_{N}=\left[\sum_{i=1}^{3} \sum_{j=1}^{3} L_{i j}^{\prime}\right] \frac{d}{d t} i_{1}
$$

or, in compact form,

$$
v_{1-3}-3 v_{N}=L_{11}^{\prime \prime} \frac{d}{d t} i_{1}
$$

This process can be generalized to any number of turns per section as illustrated in Figure 3 . Note that the reduction process is simply the addition of elements in the matrix $L^{\prime}$ by blocks. Equation (1) would become

$$
\left[\begin{array}{c}
v_{1}-w_{1} v_{N} \\
v_{2}-w_{2} v_{N} \\
\vdots \\
v_{M}-w_{M} v_{N}
\end{array}\right]=\left[\begin{array}{cccc}
L_{11}^{\prime \prime} & L_{12}^{\prime \prime} & \cdots & L_{1 M}^{\prime \prime} \\
L_{21}^{\prime \prime} & L_{22}^{\prime \prime} & \cdots & L_{2 M}^{\prime \prime} \\
\vdots & \vdots & & \vdots \\
L^{\prime \prime} & L_{M 2}^{\prime \prime} & \cdots & L_{M M}^{\prime \prime}
\end{array}\right] \frac{d}{d t}\left[\begin{array}{c}
i_{1} \\
i_{2} \\
\vdots \\
i_{M}
\end{array}\right]
$$

where

$$
M=\text { number of sections }
$$

$w_{i}=$ number of lumped turns in section $i$

$N=w_{1}+w_{2}+\cdots+w_{M}+1$

The matrix $\mathbf{L}^{\text {" }}$ of equation (9) is a modified (reduced) version of the loop inductance matrix $L^{\prime}$. The new loops involve several simple loops in series; the definition of a simple loop was given in [1].

\section{COMPLETE MAGNETIC MODEL}

The model for the windings of a transformer is given by equation (9). This model is calculated from leakage inductances assuming that the iron-core is perfect. The model can predict accurately the transfer characteristics of the transformer but it assumes that $\mu=\infty$, so that the iron-core (i.e. the magnetizing current) is not properly represented. On the other hand, iron-cored devices have traditionally been modeled using the principle of duality with the disadvantages noted in the Introduction. In this section we show how the two models can be used together so that their drawbacks are eliminated.

\section{Test Turns}

For the purpose of interconnecting the two models we require test turns that measure the leakage inductance and simultaneously the leakage flux. This can be achicved if the test tums are very thin. To measure the total leakage flux per transformer leg, we use two test turns as shown in Figure 4.

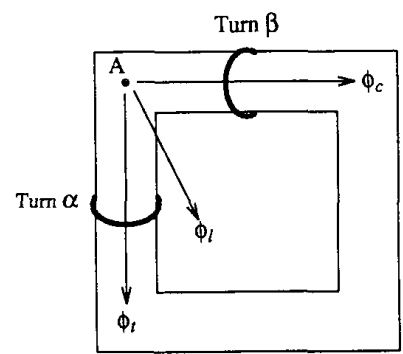

Figure 4. Test tums to measure the leakage flux 
In Figure 4 tum $\alpha$ links the leg flux while tum $\beta$ links only the yoke flux. Thus the flux difference $\phi_{\alpha}-\phi_{\beta}$ is the leakage flux. Test turns such as $\alpha$ and $\beta$ can be used to define sections of the iron-core for lumping leakage fluxes into a single node. For example, the leakage flux between our two test tums is lumped to node A (see Figure 4).

We cannot simulate tum $\beta$ around the yoke with our image method as the required geometry is axisymmetric. We can, however, choose another location for this test tum so that it links the same flux; see Figure 5. If we consider that the leakage flux is axial, or almost axial, we can estimate the radius of tum $\beta$ as half the distance between the centerlines of the legs of the transformer.

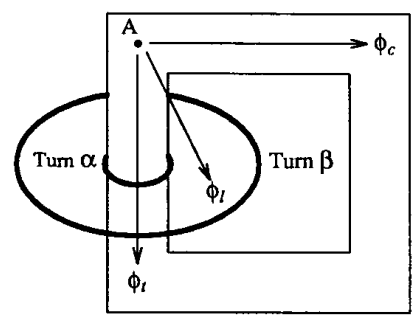

Figure 5. Altemative test turn

The Augmented Matrix $\mathbf{L}_{a}^{\prime}$

We can compute an augmented matrix $\mathbf{L}_{a}^{\prime}$ including the $\beta$ test tum, using the test tum $\alpha$ as the reference and following the process established in reference [1]. Then, we have

$$
\left[\begin{array}{c}
v_{1}-v_{\alpha} \\
v_{2}-v_{\alpha} \\
\vdots \\
v_{N}-v_{\alpha} \\
v_{\beta}-v_{\alpha}
\end{array}\right]=\left[\begin{array}{ccccc}
L_{11}^{\prime} & L_{12}^{\prime} & \cdots & L_{1 N}^{\prime} & L_{1 \beta}^{\prime} \\
L_{21}^{\prime} & L_{22}^{\prime} & \cdots & L_{2 N}^{\prime} & L_{2 \beta}^{\prime} \\
\vdots & \vdots & & \vdots & \vdots \\
L_{M 1}^{\prime} & L_{M 2}^{\prime} & \cdots & L_{N N}^{\prime} & L_{N \beta}^{\prime} \\
L_{\beta 1}^{\prime} & L_{\beta 2}^{\prime} & \cdots & L_{\beta N}^{\prime} & L_{\beta \beta}^{\prime}
\end{array}\right] \quad \frac{d}{d t}\left[\begin{array}{c}
i_{1} \\
i_{2} \\
\vdots \\
i_{N} \\
i_{\beta}
\end{array}\right]
$$

After reducing (lumping) turns in series we have an equation for sections

$$
\left[\begin{array}{c}
v_{1}-w_{1} v_{\alpha} \\
v_{2}-w_{2} v_{\alpha} \\
\vdots \\
v_{M}-w_{M} v_{\alpha} \\
v_{\beta}-v_{\alpha}
\end{array}\right]=\left[\begin{array}{ccccc}
L_{11}^{\prime \prime} & L_{12}^{\prime \prime} & \cdots & L_{1 M}^{\prime \prime} & L_{1 \beta}^{\prime \prime} \\
L_{21}^{\prime \prime} & L_{22}^{\prime \prime} & \cdots & L_{2 M}^{\prime \prime} & L^{\prime \prime}{ }_{2 \beta} \\
\vdots & \vdots & & \vdots & \vdots \\
L_{M 1}^{\prime \prime} & L_{M 2}^{\prime \prime} & \cdots & L_{M M}^{\prime \prime} & L_{M \beta}^{\prime \prime} \\
L_{\beta 1}^{\prime \prime} & L_{\beta 2}^{\prime \prime} & \cdots & L_{\beta M}^{\prime} & L_{\beta \beta}^{\prime \prime}
\end{array}\right] \frac{d}{d t}\left[\begin{array}{c}
i_{1} \\
i_{2} \\
\vdots \\
i_{M} \\
i_{\beta}
\end{array}\right]
$$

In compact form, (11) becomes

$$
\mathbf{v}_{a}-\mathbf{w}_{a} e_{a}=\mathbf{L}^{\prime \prime} \frac{d}{d t} \mathbf{i}_{a}
$$

where

$$
\begin{gathered}
\mathbf{v}_{a}=\left[\mathbf{v}^{T}, e_{a}\right]^{T}=\left[v_{1}, v_{2}, \cdots, v_{M}, e_{y_{1}}\right]^{T} \\
\mathbf{i}_{a}=\left[\mathbf{i}^{T}, i_{\beta}\right]^{T}=\left[i_{1}, i_{2}, \cdots, i_{M}, i_{\beta}\right]^{T} \\
\mathbf{w}_{a}=\left[\mathbf{w}^{T}, 1\right]^{T}=\left[w_{1}, w_{2}, \cdots, w_{M}, 1\right]^{T}
\end{gathered}
$$

We have substituted $e_{a}$ for $v_{\alpha}$ and $e_{y_{1}}$ for $v_{\beta}$ in equation (12) to make clearer that they represent voltages due to the iron-core. There are two reasons for taking tum $\alpha$ as the reference. First, it is a fictitious turn, and thus equation (12) includes the voltages and currents of all actual turns as variables. Second, its flux is the common flux that all turns link: therefore, the voltage of turn $\alpha$ represents the common voltage $e_{\alpha}$ of all turns. Thus equation (12), rewritten as

$$
\mathrm{v}_{a}=\mathrm{w}_{a} e_{a}+\mathrm{L}_{a}^{\prime \prime} \frac{d}{d t} \mathrm{i}_{a}
$$

shows that the voltage of each section (or tum) has a common component $e_{a}$ due to the flux through the leg and a component due to the flux in the air.
We can include in equation (13) the resistance of the conductors, as follows

$$
\mathbf{v}_{a}=\mathbf{w}_{a} e_{a}+\mathbf{R}_{a} \mathbf{i}_{a}+\mathbf{L}^{\prime \prime} \frac{d}{d t} \mathbf{i}_{a}
$$

Here $\mathbf{R}_{a}$ is a diagonal matrix whose elements are the sum of the resistances of the turns in each section.

Note that all inductance matrices are calculated with the use of the image method based on axisymmetric geometry. As a consequence, the magnetic effects represented by these matrices have mutual coupling only with the tums (or windings) that are wound on the same leg. The image method does not permit to include the (small) mutual inductances due to leakage fluxes between coils wound on different legs.

\section{Model from Duality}

Applying the principle of duality to a three phase (three-legged) transformer, we obtain the electric equivalent circuit shown in Figure 6 described in references [2]-[6]. This equivalent circuit consists of five nonlinear inductors, $L_{a}, L_{b}, L_{c}, L_{y_{1}}$ and $L_{y_{2}}$, that represent the flux in the iron-core. It has three linear (positive) inductors that represent the leakage flux, $L_{a_{i}}, L_{b_{l}}$ and $L_{c_{i}}$. The circuit also has a number of negative (linear) inductors in series with the terminals (only two terminals per leg are shown), $L_{a_{1}} \cdots L_{c_{2}}$.

For the nonlinear inductor on leg $a$ (if the current through it is $i_{\alpha}$ ), we have

$$
e_{a}=\frac{d \phi}{d t} \quad \phi=\phi\left(i_{\alpha}\right)
$$

from where we get

$$
e_{a}=L_{a}\left(i_{\alpha}\right) \frac{d}{d t} i_{\alpha}
$$

where

$$
L_{a}\left(i_{\alpha}\right)=\frac{d}{d i_{\alpha}} \phi\left(i_{\alpha}\right)
$$

A similar equation can be obtained for each nonlinear inductor.

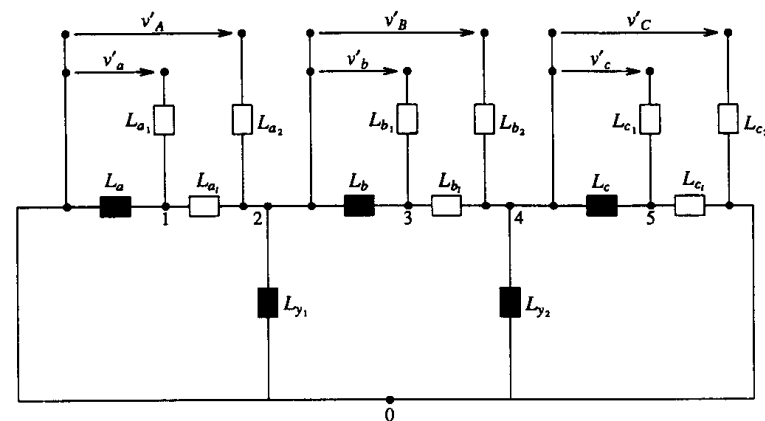

Figure 6. Application of the principle of duality

\section{Complete Model}

The interfaces between the model based on leakage inductances and the model from duality are the test turns $\alpha$ and $\beta$ on each leg. Turn $\alpha$ can be considered to be the connection to the leg inductor $\left(L_{a}\right.$, for example) while turn $\beta$ is used to connect the model to the yoke inductor (such as $L_{y_{1}}$ ). In Figure 7 we show the complete model for one leg.

We can see that the sum of currents entering block $L^{\prime \prime}{ }_{a}$ has to be zero:

$$
\sum_{k=1}^{N} w_{k} i_{a_{k}}-i_{\alpha}-i_{\beta}=0
$$

The sum of $i_{\alpha}$ and $i_{\beta}$ is the magnetizing current. Thus, from (17), we have

$$
\sum_{k=1}^{N} w_{k} i_{a_{k}}=i_{\alpha}+i_{\beta}=i_{m a g}
$$




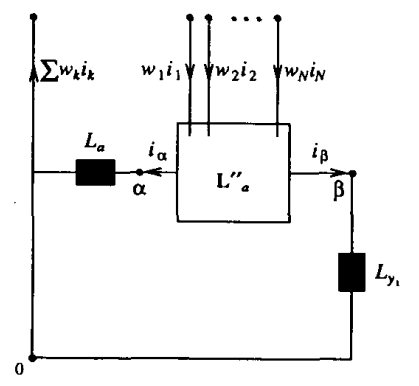

Figure 7. Complete model for one leg of a transformer

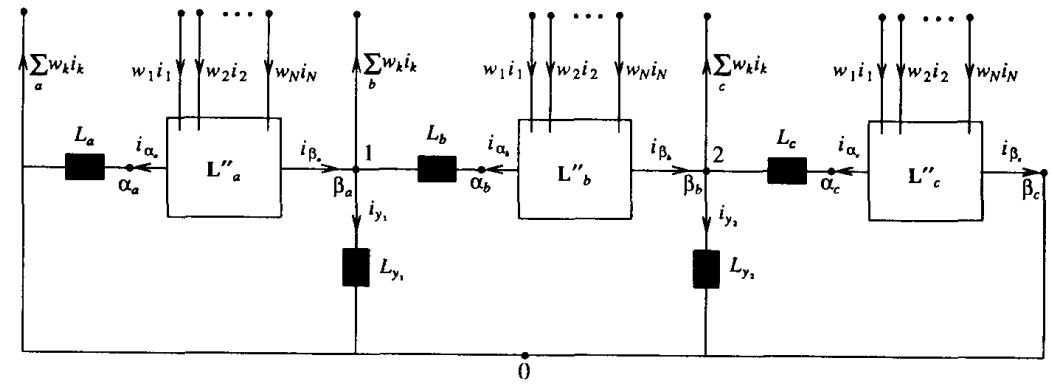

Figure 8. Complete model for a three-legged transformer
We have a total of $N+4$ unknowns: $N$ voltages or currents for the sections or turns, plus $e_{a}, e_{b}, i_{\alpha}$, and $i_{\beta}$. The equations for the complete model are the $N+1$ voltage equations given by equation (14), and two nonlinear equations, as (16), for the iron-core elements, and the KCL equation (17). Thus, we have a proper set of equations, some of which are nonlinear.

For a three-legged transformer we would have a model as shown in Figure 8 . This model can be obtained by substituting an $\mathbf{L}^{\text {" block (see }}$ Figure 7) for the inductance network in Figure 6. It is important to note that all the equivalent circuits derived from the principle of duality are only electric representations of magnetic circuits. These circuits are normally referred to a common number of tums (in our models this number is one). In all cases (Figures 6,7 and 8), the actual electric connections have to be done extemally. Also, the capacitances are to be connected to the terminals of the turns (or sections) extemally to the magnetic model.

The equations describing the three-legged transformer are

$$
\begin{aligned}
& \mathbf{v}_{a}=\mathbf{w}_{a} e_{a}+\mathbf{R}_{a} \mathbf{i}_{a}+\mathbf{L}^{\prime \prime}{ }_{a} \frac{d}{d t} \mathbf{i}_{a} \\
& \mathbf{v}_{b}=\mathbf{w}_{b} e_{b}+\mathbf{R}_{b} \mathbf{i}_{b}+\mathbf{L}^{\prime \prime}{ }_{b} \frac{d}{d t} \mathbf{i}_{b} \\
& \mathbf{v}_{c}=\mathbf{w}_{c} e_{c}+\mathbf{R}_{c} \mathbf{i}_{c}+\mathbf{L}^{\prime \prime}{ }_{c} \frac{d}{d t} \mathbf{i}_{c} \\
& e_{a}=L_{a}\left(i_{\alpha_{a}}\right) \frac{d}{d t} i_{\alpha_{a}} \\
& e_{b}=L_{b}\left(i_{\alpha_{b}}\right) \frac{d}{d t} i_{\alpha_{b}} \\
& e_{c}=L_{c}\left(i_{\alpha_{c}}\right) \frac{d}{d t} i_{\alpha_{c}} \\
& e_{y_{1}}=L_{y_{1}}\left(i_{y_{1}}\right) \frac{d}{d t} i_{y_{1}} \\
& e_{y_{2}}=L_{y_{2}}\left(i_{y_{2}}\right) \frac{d}{d t} i_{y_{2}} \\
& \sum_{k=1}^{N} w_{k} i_{a_{k}}-i_{\alpha_{a}}-i_{\beta_{a}}=0 \\
& \sum_{k=1}^{N} w_{k} i_{b_{k}}-i_{\alpha_{b}}-i_{\beta_{b}}=0 \\
& \sum_{k=1}^{N} w_{k} i_{c_{k}}-i_{\alpha_{k}}-i_{\beta_{e}}=0 \\
& \sum_{k=1}^{N} w_{k} i_{b_{k}}-i_{\alpha_{b}}-i_{\beta_{a}}+i_{y_{1}}=0 \\
& \sum_{k=1}^{N} w_{k} i_{b_{k}}-i_{\alpha_{k}}-i_{\beta_{b}}+i_{y_{2}}=0
\end{aligned}
$$

The last two equations (equations (21)) are very important conceptually since they represent the fact that the sum of the currents inside a window is zero. These two equations correspond to the KCL in the nodes 1 and 2 of Figure 8 and represent, in the electric equivalent circuit, the mmf's (of the magnetic circuit) around the windows.

Equations (18) to (21) form a fully determined set of equations for a three phase (three-legged) transformer (magnetic model).

\section{CALCULATION OF TRANSIENTS} Inductive Model

For the single legged transformer described above (by equations (14), (16) and (17)) we obtain the transient model by applying the trapezoidal rule of integration. From equation (14) we have

$$
\mathbf{R}_{r H} \mathbf{i}_{a}+\mathbf{w}_{a} e_{a}=\mathbf{v}_{a}+\mathbf{v}_{a}^{\text {hist }}
$$

and integrating equation (16) we get

$$
i_{\alpha}=G_{\alpha} e_{\alpha}+i_{\alpha}^{h i s t}
$$

The superscript hist stands for history and accounts for the previous values of current and voltage in the circuit. Equation (22) represents a Thevenin equivalent for the windings, and equation (23) models the iron-core with a Norton equivalent. Note that in equation (23) the conductance $G$ is a function of the current through the nonlinear inductor The derivation of these equations can be found in Appendix 1 .

These two equations form together with the KCL equation (equation (17)) the transient model for the magnetic part of one leg of a transformer. The equations have to be solved by an iterative method since they contain some nonlinearities. When the core is not saturated (or lightly saturated), the currents $i_{\alpha}, i_{\beta}$ through the inductors representing the iron are small compared with the currents in the actual turns. This allows to transfer them to the history vector as a first approximation, and then solve the equations iteratively. This gives fast convergence and more importantly, permits to decouple the magnetizing (nonlinear) equations from the leakage equations. A more detailed discussion on convergence is presented in Appendix 2. We can obtain a first approximation if we estimate the magnetizing current $i_{m a g}=i_{\alpha}+i_{\beta}$ in equation (17a) and transfer it to the history as $i_{\text {mag }}^{\text {hist }}$. Then we get

$$
\sum_{k=1}^{N} w_{k} i_{a_{k}}=i_{m a g}^{\text {hist }}
$$

After one iteration we can update this value. Combining equations (22) with (24) we form

$$
\left[\begin{array}{cc}
\mathbf{R}_{T H} & \mathbf{w}_{a} \\
\mathbf{w}_{a}^{T} & 0
\end{array}\right]\left[\begin{array}{c}
\mathbf{i}_{a} \\
e_{a}
\end{array}\right]=\left[\begin{array}{c}
\mathbf{v}_{a} \\
0
\end{array}\right]+\left[\begin{array}{c}
\mathbf{v}_{a \text { mag }}^{\text {hist }} \\
i_{\text {mag }}^{\text {hist }}
\end{array}\right]
$$

In compact form, equation (25) is

$$
\mathbf{R}_{\text {aug }} \mathbf{i}_{\text {aug }}=\mathbf{v}_{\text {aug }}+\mathbf{v}_{\text {aug }}^{\text {hist }}
$$

For the iron-core we have the following relations: from equation (23),

$$
i_{\alpha}=G_{\alpha} e_{\alpha}+i_{\alpha}^{\text {hist }}
$$

and, similarly, for the inductor representing the yoke,

$$
i_{\beta}=G_{\beta} e_{y_{1}}+i_{\beta}^{\text {hist }}
$$

Equation (26) is a hybrid system of linear equations that can be solved (after estimating an initial value for $i_{\alpha}$ and $i_{\beta}$ ) in combination with the extemal circuit (including the capacitances) to get the first approximation for $i_{1}, i_{2}, \cdots, i_{N}, v_{1}, v_{2}, \cdots, v_{N}, i_{\alpha}, i_{\beta}, e_{a}$, and $e_{y_{1}}$. Later, we use equa- 
tions (27) to update $i_{\alpha}$ and $i_{\beta}$ for the next iteration. Thus we have two decoupled steps in the iterative solution of the equations at each time step. Note that in equation (25) the matrix remains constant during the iterative process and only the history terms change. In equations (27) the coefficients $G_{\alpha}$ and $G_{\beta}$ do vary, but they are scalars.

For a three-phase transformer we would have to follow the same procedure as described above, integrating equations (18) and (19) to obtain a Thevenin and a Norton equivalent circuit per leg. These Thevenin equivalents are coupled through the iron-core (variables $e_{y_{1}}$ and $e_{y_{2}}$ are common to two legs). The resulting equation has the same form as equation (25), but instead of the w vector, we have matrices:

$$
\left[\begin{array}{cc}
\mathbf{R}_{T H} & \mathbf{W} \\
\mathbf{W}^{T} & \mathbf{0}
\end{array}\right]\left[\begin{array}{l}
\mathbf{i} \\
\mathbf{e}
\end{array}\right]=\left[\begin{array}{l}
\mathbf{v} \\
\mathbf{0}
\end{array}\right]+\left[\begin{array}{l}
\mathbf{v}_{\text {mag }}^{\text {hist }} \\
\mathbf{i}_{\text {mag }}^{\text {hist }}
\end{array}\right]
$$

where

$$
\begin{aligned}
& \mathbf{R}_{T H}=\left[\begin{array}{lll}
\mathbf{R}_{T H a} & & \\
& \mathbf{R}_{T H b} & \\
& & \mathbf{R}_{T H c}
\end{array}\right] \\
& \mathbf{W}=\left[\begin{array}{lllll}
\mathbf{w} & & & & \\
1 & & & -1 & \\
& \mathbf{w} & & & \\
& 1 & & -1 & -1 \\
& & \mathbf{w} & & \\
& & 1 & & -1
\end{array}\right] \\
& \mathbf{i}=\left[\mathbf{i}_{a}^{T}, \mathbf{i}_{b}^{T}, \mathbf{i}_{c}^{T}\right]^{T} \\
& \mathbf{e}=\left[e_{a}, e_{b}, e_{c}, e_{y_{1}}, e_{y_{2}}\right]^{T} \\
& \mathbf{v}=\left[\mathbf{v}_{a}^{T}, \mathbf{v}_{b}^{T}, \mathbf{v}_{c}^{T}\right]^{T} \\
& \mathbf{v}_{\text {mag }}^{\text {hist }}=\left[\mathbf{v}_{a \text { mag }}^{\text {hist } T}, \mathbf{v}_{b \text { mag }}^{\text {hist } T}, \mathbf{v}_{c \text { mag }}^{\text {hist } T}\right]^{T} \\
& \mathbf{i}_{\text {mag }}^{\text {hist }}=\left[i_{\alpha_{0}}, i_{\alpha_{b}}, i_{\alpha_{c}}, i_{y_{1}}, i_{y_{2}}\right]^{T}
\end{aligned}
$$

Note that in $\mathbf{i}_{a}, \mathbf{i}_{b}$ and $\mathbf{i}_{c}$ the currents of the $\beta$ turns are included. Similarly, the corresponding voltages for the $\beta$ tums are also included in $\mathbf{v}_{a}$, $\mathbf{v}_{a \text { mag }}^{\text {hist }}$, etc.

The complete model includes the five nonlinear equations for the iron-core, given by

$$
\begin{aligned}
& i_{\alpha_{a}}=G_{\alpha_{a}} e_{a}+i_{\alpha_{a}}{ }^{\text {hist }} \\
& i_{\alpha_{b}}=G_{\alpha_{b}} e_{b}+i_{\alpha_{b}}{ }^{\text {hist }} \\
& i_{\alpha_{a}}=G_{\alpha_{c}} e_{c}+i_{\alpha_{c}}{ }^{\text {hist }} \\
& i_{y_{1}}=G_{y_{1}} e_{y_{1}}+i_{y_{1}}{ }^{\text {hist }} \\
& i_{y_{2}}=G_{y_{2}} e_{y_{2}}+i_{y_{2}}{ }^{\text {hist }}
\end{aligned}
$$

These models can be interfaced with the capacitive networks and with the external circuit and we can include the capacitances between different legs.

The idea of decoupling is very important as it permits to reduce the order of the computational problem and to handle separately the linear and nonlinear parts of the transformer model. This is reflected in Figure 9. The figure shows the flow of information between the two parts of the model.

\section{Terminal Model} get

Integrating the capacitive equation (2) with the trapezoidal rule, we

$$
\mathbf{i}_{C \text { node }}=\mathbf{G}_{C \text { node }} \mathbf{v}_{\text {node }}+\mathrm{i}_{C \text { node }}^{\text {hisl }}
$$

The expressions of $\mathrm{G}_{C}$ and $\mathbf{i}_{C}^{\text {hist }}$ are given in Appendix 1 .

Equation (30) is in the nodal reference frame while equation (26) is in the branch reference frame. In order to solve them together, we convert equation (26) to the nodal reference frame by the use of a powerinvariant transformation defined as

$$
\begin{aligned}
\mathbf{v}_{\text {aug }} & =\mathbf{A} \quad \mathbf{v}_{\text {node }} \\
\mathbf{i}_{\text {L node }} & =\mathbf{A}^{T} \mathbf{i}_{\text {aug }}
\end{aligned}
$$

where $\mathbf{A}$ is the node-element incidence matrix. This yields

$$
\mathbf{i}_{L \text { node }}=\mathbf{G}_{L \text { node }} \mathbf{v}_{\text {node }}+\mathbf{i}_{L \text { node }}^{\text {hist }}
$$

The expressions of the new matrices and vectors can be found in Appendix 1. Adding equations (30) and (32) and dropping the subscripts for nodes, we obtain

$$
\mathbf{i}=\mathbf{G} \mathbf{v}+\mathbf{i}^{\text {hist }}
$$

It is convenient to reorder the nodes in the above equation to have the external nodes at the top of the matrix $G$. Then we obtain a Norton equivalent circuit for the external nodes by partitioning equation (33) as

$$
\left[\begin{array}{l}
\mathbf{i}_{e} \\
0
\end{array}\right]=\left[\begin{array}{ll}
\mathbf{G}_{e e} & \mathbf{G}_{e i} \\
\mathbf{G}_{i e} & \mathbf{G}_{i i}
\end{array}\right]\left[\begin{array}{l}
\mathbf{v}_{e} \\
\mathbf{v}_{i}
\end{array}\right]+\left[\begin{array}{l}
\mathbf{i}_{e}^{h i s t} \\
\mathbf{i}_{i}^{h i s t}
\end{array}\right]
$$

from which we obtain

$$
\mathbf{v}_{i}=-\mathbf{G}_{i i}^{-1} \mathbf{G}_{i e} \mathbf{v}_{e}-\mathbf{G}_{i i}^{-1} \mathbf{i}_{i}^{h i s t}
$$

and

$$
\mathbf{i}_{e}=\mathbf{G}_{e q} \mathbf{v}_{e}+\mathbf{i}_{e q}^{\text {hist }}
$$

See Appendix 1 for the expressions of the new variables.

Equation (36) should be solved at each integration step in conjunction with the Norton equivalent equations of the external system. Subsequently, we can solve for the voltages at the internal nodes and for the currents through the branches. Finally, we solve for the voltages and currents in the fictitious tums ( $\alpha$ and $\beta$ ) and we iterate until we get convergence for each integration step. Note that, again, all matrices remain constant during the iterative process and only the history vectors change.

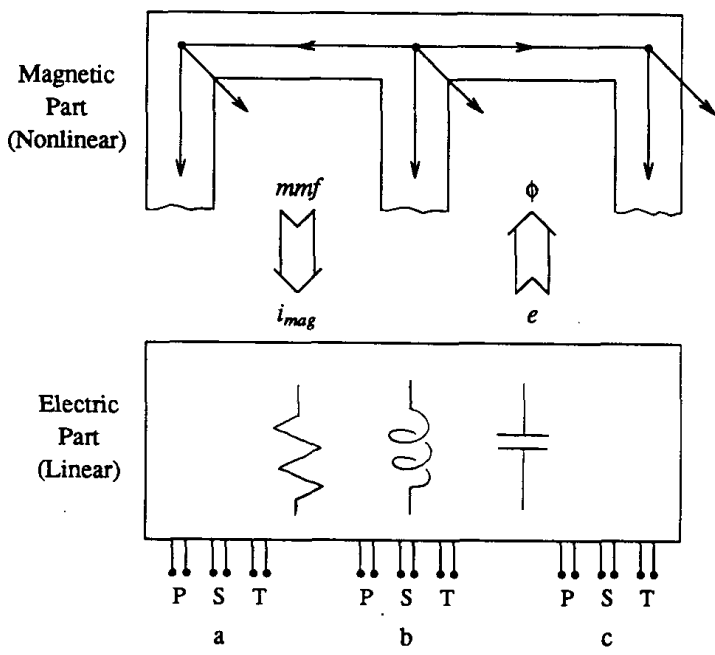

Figure 9. Decoupled transformer model

\section{FREQUENCY RESPONSE}

For the simulation of the frequency response we set $\frac{d}{d t}=j \omega$. We could follow similar steps as in the case of transients presented above and would obtain an equivalent admittance matrix at the transformer terminals, given by

$$
\mathrm{I}_{e}=\mathrm{Y}_{e q} \mathrm{~V}_{e}
$$

We interface equation (37) with the external circuit and then we solve iteratively towards the inside of the transformer since we have some nonlinear equations for the iron-core. As in the case for transients, all matrices remain constant. The nonlinearities are reflected, in this case, only in the input vector (for example, $\mathbf{V}_{e}$ in equation (37)) 
RESULTS

Frequency Response

We have used the procedure described in reference [1] (bucking test) to obtain the driving point admittance for different frequencies and for different degrees of reduction of the model. The 236 tum (per leg) transformer, described in Appendix 3, was reduced to 6,14, 28 and 52 sections per leg. The test (or series) leakage inductance (at low frequencies) is obtained using a process similar to that described in Appendix 1 of [1]. The leakage inductances were exactly the same (up to 5 digits at $60 \mathrm{~Hz}$ ) for all cases: $L_{\text {leak }}=4.3 \times 10^{-4}$. From tests at $60 \mathrm{~Hz}$ we get: $L_{\text {leak }}=4.5 \times 10^{-4}(\approx 5 \%$ error $)$. In Figure 7 of [1] we have shown the variation of the input admittance with frequency when the transformer is short-circuited. The results are in good agreement with measurements up to a frequency of $700 \mathrm{kHz}$.

\section{Transients}

We have simulated various transients for the transformer described in Appendix 3. The first case is the energization of the transformer from a d.c. voltage source $(V=1)$ in series with a resistance $(R=100 \Omega)$, when the secondary is in open circuit. The 236 turns per leg are reduced to four sections of 59 turns each.

In Figure $10 \mathrm{a}$, we present the resulting (fast) transient that corresponds to the charging of the capacitances and the oscillations between the capacitances and the leakage inductances. The frequency of oscillation, of the order of $1 \mathrm{MHz}$, matches approximately the value calculated from $v=1 /\left(\sqrt{L_{\text {leak }} C_{e q}}\right)$ with $C_{e q} \approx 10^{-10} \mathrm{~F}$ and $L_{\text {leak }} \approx 10^{-4} \mathrm{H}$

Once the fast oscillations have been damped, we have the slow transient of the R,L series circuit, shown in Figure 10b. We can see that the voltage tends to zero exponentially with a time constant $T=L_{\text {mag }} / R$. With $R=100 \Omega$ and $L_{\text {mag }} \approx 1 \mathrm{H}$ we have $T \approx 0.01 \mathrm{~s}$ Note that the fast oscillations do not appear in the simulation of this case due to the large integration time step.

The second example represents the chopping of the magnetizing current. We first energize the transformer (using the same circuit as before) and after $100 \mu \mathrm{s}$ we disconnect the primary from the source. As expected, we have an oscillatory voltage. This is shown in Figure 11. The frequency of oscillation of this transient corresponds approximately to the frequency estimated with simplified calculations, using this time the inductance $L_{\text {mag }}$.

The decoupled solution method requires iterations within each time step, as described before and illustrated in Figure 9. The number of these iterations is typically 3 to 5 in the computations we have performed, for a tolerance of $10^{-5}$

\section{CONCLUSIONS}

The paper has described a reduced order model for the transformer windings, suitable for the study of high frequency transients. The model is based on the turn-to-turn information obtained in the efficient way shown in reference [1]. A methodology for reducing high order models was presented in detail. The winding model is interfaced with the ironcore model using the principle of duality and test turns. The model has been validated with both frequency and time domain simulations. The terminal transient model is a Norton equivalent that can be interfaced with a power systems transients program.

\section{ACKNOWLEDGEMENTS}

Financial support by the Natural Sciences and Engineering Research Council of Canada is gratefully acknowledged. The first author wishes to express his sincere gratitude to the National Council of Science and Technology of Mexico (CONACYT) and to the National Polytechnic Institute of Mexico for the financial support of his studies at the University of Toronto.

\section{REFERENCES}

[1] F. de Leon and A. Semlyen, "Efficient Calculation of Elementary Parameters of Transformers", paper accepted for presentation at the IEEE/PES 1991 Winter Meeting.

[2] E. Colin Cherry, "The Duality between Interlinked Electric and Magnetic Circuits and the Formation of Transformer Equivalent Circuits", Proc. of the Physical Society, Vol. (B) 62, Feb. 1949, pp. 101-111.

[3] G.R. Slemon, "Equivalent Circuits for Transformers and Machines

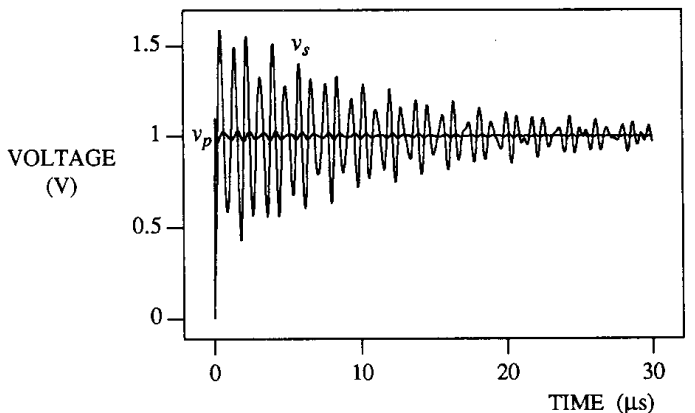

Figure 10a. Transient voltages during energization of the transformer (primary and secondary voltages)

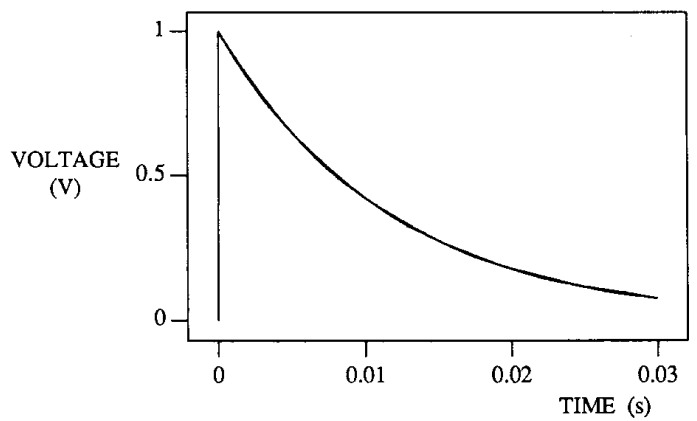

Figure $10 \mathrm{~b}$. Transient voltages during energization of the transformer (primary and secondary voltages)

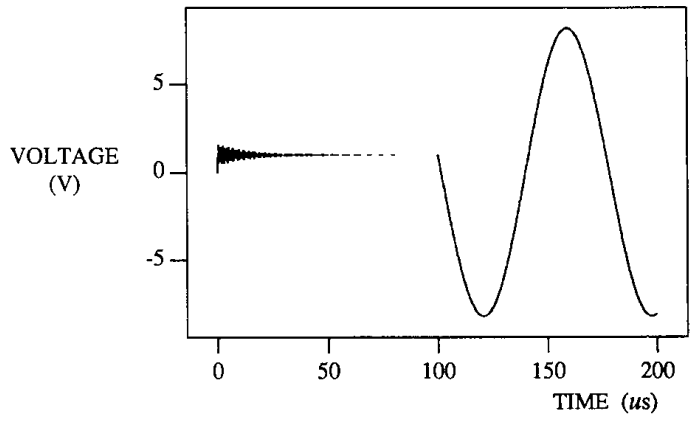

Figure 11. Transformer energization and disconnection at $t=100 \mu \mathrm{s}$ (primary and secondary voltages)

Including Nonlinear Effects", Proc. IEE, Part IV, Vol. 100, 1953, pp. 129-143.

[4] H. Edelmann, "Anschauliche Ermittlung von Transformator-Ersatzschaltbildern", Arch. elektr. Ubertragung, Vol. 13, 1959, pp. 253-261.

[5] L. Krähenbühl, B. Kulicke, and A. Webs "Simulationsmodell eines Mehrwicklungstransformators zur Untersuchung von Sättigungsvorgängen", Siemens Forschung und Entwicklungs Bericht, Band 12 (1983), Nr 4, pp. 232-235.

[6] C.M. Arturi, "Transient Simulation and Analysis of a Three-Phase StepUp Transformet Following an Out-Phase Synchronization", IEEE/PES Winter Meeting 1990, paper No. 90 WM 225-3 PWRD.

[7] L.F. Blume, et al., "Transformer Engineering", Second Edition, John Wiley and Sons, 1951.

[8] J.H. McWhirter, C.D. Fahrnkopf, and J.H. Steele, "Determination of Impulse Stresses within Transformer Windings by Computers", AIEE Transactions on Power Apparatus and Systems, February 1957, pp. 1267. 1274

[9] R.B. Shipley, D. Coleman, and C.F. Watts, "Transformer Circuits for Digital Studies", AIEE Transactions Pt. III (Power Apparatus and Systems), Vol-81, February 1963, pp. 1028-1031. 
[10] H. W. Dommel, "Transformer Models in the Simulation of Electromagnetic Transients", Proc. 5th Power Systems Computation Conference, 1975, Sept. 1-5, Paper 3.1/4

[11] R.C. Dugan, R. Gabrik, J.C. Wright, and K.W. Patten, "Validated Techniques for Modeling Shell-Form EHV Transformers", IEEE Transactions on Power Delivery, Vol-4, No. 2, April 1989, pp. 1070-1078.

[12] DJ. Wilcox, M. Conlon, and W.G. Hurley, "Calculation of Self and Mutual Impedances for Coils on Ferromagnetic Cores", IEE Proceedings, Vol. 135, Pt. A, No. 7, September 1988, pp. 470-476.

[13] D.J. Wilcox, W.G. Hurley, and M. Conlon, "Calculation of Self and Mutual Impedances Between Sections of Transformer Windings", IEE Proceeding, Vol. 136, Pt. C, No. 5, September 1989, pp. 308-314.

\section{APPENDICES}

Appendix 1. Definition of Some Variables

First we present the application of the trapezoidal rule of integration. The Thevenin equivalent circuit representing the winding model for a one-legged transformer (derived from the trapezoidal rule of integration) is given by equation (22) where

$$
\begin{gathered}
\mathbf{R}_{T H}=\mathbf{R}_{a}+\frac{2}{\Delta t} \mathbf{L}_{a}^{\prime \prime} \\
\mathbf{v}_{a}^{\text {hist }}=\mathbf{v}_{a}^{\text {old }}-\mathbf{w}_{a} e_{a}^{\text {old }}-\mathbf{R}_{T H} \mathrm{i}_{a}^{\text {old }}
\end{gathered}
$$

For the core model we get the Norton equivalent of equation (23) where

$$
\begin{gathered}
G_{\alpha}=\frac{\Delta t}{2} L_{\alpha}\left(i_{\alpha}\right)^{-1} \\
i_{\alpha}^{\text {hist }}=i_{\alpha}^{\text {old }}+G_{\alpha} e_{\alpha}^{\text {old }}
\end{gathered}
$$

The superscript old stands for previous value (or initial condition for the firs step). Note that in equation (23) the conductance $G_{\alpha}$ is a function of the current through the nonlinear inductor.

The Norton equivalent for the capacitors is given in equation (30) where

$$
\begin{gathered}
\mathbf{G}_{C \text { node }}=\frac{2}{\Delta t} \mathbf{C}_{\text {node }} \\
\mathbf{i}_{C \text { node }}^{\text {hist }}=\mathbf{G}_{C_{\text {node }}} \mathbf{v}_{\text {node }}^{\text {old }}+\mathbf{i}_{C \text { node }}^{\text {old }}
\end{gathered}
$$

Below are the expressions of some variables given in the main text. In equation (32) we have

$$
\begin{aligned}
& \mathbf{G}_{L_{\text {node }}}=\mathbf{A}^{T} \mathbf{R}_{\text {aug }}^{-1} \mathbf{A} \\
& \mathbf{i}_{L_{\text {node }}^{\text {hist }}}=\mathbf{A}^{T} \mathbf{R}_{\text {akg }}^{-1} \mathbf{v}_{\text {aug }}^{\text {hist }}
\end{aligned}
$$

In equation (36) we have

$$
\begin{gathered}
\mathbf{G}_{e q}=\mathbf{G}_{e e}-\mathbf{G}_{e i} \mathbf{G}_{i i}^{-1} \mathbf{G}_{i \epsilon} \\
\mathbf{i}_{e q}^{\text {hist }}=-\mathbf{G}_{e i} \mathbf{G}_{i i j}^{-1} \mathbf{i}_{i}^{h i s t}+\mathbf{i}_{e}^{h i s t}
\end{gathered}
$$

\section{Appendix 2. Convergence Properties of the Nonlinear Equations}

The model of the transformer (neglecting the resistance) is given by equations (13), (16) and (17). Using a new set of variables for the derivative of the currents, defined by:

$$
i^{\prime}=\frac{d}{d t} i
$$

we can rewrite the equations as:

$$
\begin{gathered}
\mathrm{v}_{a}=w_{a} e_{a}+L^{\prime \prime}{ }_{a} i_{a}^{\prime} \\
e_{a}=L_{\alpha}\left(i_{\alpha}^{\prime}\right) i_{\alpha}^{\prime} \\
e_{y_{1}}=L_{\beta}\left(i_{\beta}^{\prime}\right) i_{\beta}^{\prime} \\
\sum_{k=1}^{N} w_{k} i_{a_{k}}^{\prime}-i_{\alpha}^{\prime}-i_{\beta}^{\prime}=0 .
\end{gathered}
$$

Equation (A-2) can be partitioned as shown next:

$$
\left[\begin{array}{ll}
\mathbf{L}_{a, \alpha}^{\prime \prime} & \mathbf{L}_{a, \beta}^{\prime \prime} \\
\mathbf{L}_{\beta, a}^{\prime \prime} & L_{\beta, \beta}^{\prime \prime}
\end{array}\right]\left[\begin{array}{l}
i^{\prime} \\
i_{\beta}^{\prime}
\end{array}\right]+\left[\begin{array}{c}
\mathbf{w} \\
\mathbf{l}
\end{array}\right] e_{a}=\left[\begin{array}{c}
\mathbf{v} \\
e_{y_{1}}
\end{array}\right]
$$

We can rearrange equations (A-3), (A-4), (A-5) and (A-6) as follows:

$$
\left[\begin{array}{ccccc}
\mathbf{L}^{\prime \prime}{ }_{a, a} & \mathbf{L}^{\prime \prime}{ }_{a, \beta} & \mathbf{w} & 0 & 0 \\
\mathbf{w}^{T} & 1 & 0 & 0 & 1 \\
\mathbf{L}_{\beta, a}^{\prime \prime} & L_{\beta, \beta}^{\prime \prime} & 1 & -1 & 0 \\
0 & 0 & -1 & 0 & L_{\alpha} \\
0 & L_{\beta} & 0 & -1 & 0
\end{array}\right]\left[\begin{array}{c}
\mathbf{i}^{\prime} \\
\boldsymbol{e}_{\alpha} \\
e_{y_{1}} \\
i_{\alpha}^{\prime} \\
i_{\beta}^{\prime}
\end{array}\right]=\left[\begin{array}{c}
\mathbf{v} \\
0 \\
0 \\
0 \\
0
\end{array}\right]
$$

The iterative method used is based on the fact that the magnetizing currents $i_{\alpha}^{\prime}$ and $i_{\beta}^{\prime}$ are small. Thus we can define two groups of variables as follows:

$$
\begin{gathered}
\mathbf{x}_{1}^{T}=\left[i^{T}, e_{\alpha}, e_{y_{1}}\right]^{T} \\
\mathbf{x}_{2}^{T}=\left[i_{\alpha}^{\prime}, i_{\beta}^{\prime}\right]^{T}
\end{gathered}
$$

We partition equation (A-7) to get

$$
\begin{aligned}
& A x_{1}+B x_{2}=b_{1} \\
& C x_{1}+D x_{2} \doteq b_{2}
\end{aligned}
$$

If the converged solution is given by $x_{1}^{*}$ and $x_{2}^{*}$ and the error at iteration $k$ is given by $\varepsilon_{1}=x^{*}-x_{1}$ and $\varepsilon_{2}=x^{*}-x_{2}$, we can write the incremental versions of $(\mathrm{A}-10)$ and $(\mathrm{A}-11)$ as

$$
\begin{aligned}
& A \varepsilon_{1}+B \varepsilon_{2}=0 \\
& C \varepsilon_{1}+D \varepsilon_{2}=0
\end{aligned}
$$

Applying the fixed-point iteration method to equations (A-12) and (A-13) we get:

$$
\begin{aligned}
& \varepsilon_{1}{ }^{(k+1)}=M_{1} \varepsilon_{1}{ }^{(k)} \\
& \varepsilon_{2}{ }^{(k+1)}=M_{2} \varepsilon_{2}{ }^{(k)}
\end{aligned}
$$

where

$$
\begin{aligned}
& \mathbf{M}_{1}=\mathbf{A}^{-1} \mathbf{B ~ D}^{-1} \mathbf{C} \\
& \mathbf{M}_{2}=\mathbf{D}^{-1} \mathbf{C A}^{-1} \mathbf{B}
\end{aligned}
$$

Note that these two matrices are functions of $\varepsilon_{2}$ (the error in the magnetizing current) since matrix $D$ contains the nonlinear inductances.

To assess the convergence properties of the method, we may look at the non-zero eigenvalues of $\mathbf{M}_{1}$ or $\mathbf{M}_{2}$ (they are identical). If all $\left|\lambda_{i}\right|<1$, the method converges, and the smaller the largest eigenvalue, the faster is the convergence. For a one-legged transformer, it can be shown that there are only two non-zero eigenvalues.

For our (non-saturated) transformer, the two eigenvalues obtained are $\lambda_{1}=-5.3 \times 10^{-5}$ and $\lambda_{2}=1.34 \times 10^{-3}$. As the transformer saturates, the absolute value of the eigenvalues increases in inverse proportion to the permeability. Thus, for $\mu_{\text {sat }}=\mu / 100$ we have $\lambda_{1}=-5.3 \times 10^{-3}$ and $\lambda_{2}=0.134$. The maximum saturation degree for which the method converges is $1 /\left|\lambda_{\max }\right|$; for our example, this gives $\mu_{\text {sat }}=\mu / 746$.

\section{Appendix 3. Transformer Data}

Single phase, $2 \mathrm{kVA}, 2$ windings, $110 \mathrm{~V} / 110 \mathrm{~V}, 2$ layers per winding, 2 windings per leg, 59 turns per layer, 236 turns total per leg, square conducto $(a=3.5 \mathrm{~mm}$ insulated). The geometrical dimensions are shown in Figure 8 of [1].

Francisco de Leon was born in Mexico City, Mexico, in 1959. He received his B.Sc. degree and his M.Sc. degree (summa cum laude) from the National Polytechnic Institute of Mexico, in 1983 and 1986 respectively. From 1984 to 1987 he was a lecturer at the same institute. Currently, he is working towards his Ph.D. degree at the University of Toronto. His main interests are in the areas of transformer modeling and electromagnetic fields.

Adam Semlyen (F'87) was born and educated in Rumania where he obtained a Dipl. Ing. degree and his Ph.D. He started his carecr with an electric power utility and held an academic position at the Polytechnic Institute of Tim isoara, Rumania. In 1969 he joined the University of Toronto where he is a professor in the Department of Electrical Engineering. His research interests include the steady state and dynamic analysis of power systems, electromagnetic transients, and power system optimization. 


\section{Discussion}

C. M. Arturi, (Politecnico di Milano, Italy): I wish to congratulate Professor Semlyen and Dr. De Leon for their lucid and very timely paper. I particularly appreciated the description of the mathematical frame in which their models can be developed and the adopted solution method.

I should appreciate their comments on the following problems.

1. The authors have divided the ferromagnetic core in several non linear branches and, therefore, an accurate simulation in very high saturation conditions should be possible. I wonder if the equivalent inductance seen from the external terminals of the model corresponds to the air core inductance of the coils. Did the authors obtain any results to validate the model from this point of view?

2. Have the authors simulated their model by means of the present EMTP program? If so, can they please comment of these results compared to those obtained by the integration procedure as described in the paper?

3. Have the authors experienced the problem of the dissipative parameters to be considered in the models for high frequency transient voltage?

Manuscript received February 15, 1991
F. de Leon and A. Semlyen (University of Toronto): We wish to thank Dr. Arturi for his interest in our paper. The following are our answers to his questions.

1) The inductance seen from the external terminals corresponds in full saturation to the air core inductance since the leakage part of the model represents the flux outside of the iron core which will go into saturation. In reality, the actual flux inside the core will no longer follow an exclusively axial direction.

2) As our inductive model cannot be easily interpreted as a network of inductances we were forced to write our own code instead of using EMTP or any other simulation package. However, we have used the trapezoidal rule of intergration to get a Norton equivalent at the terminals of the transformer which is fully compatible with EMTP.

3) The frequency dependence of the losses (damping) is of prime interest to us and is included in the model of reference [A].

[A] F. de Leon and A. Semlyen, "Time Domain Modeling of Eddy Current Effects for Transformer Transients", to be presented at the IEEE/PES 1992 Winter Meeting.

Manuscript received August 9, 1991. 